Tome 38, no 23. - Novembre 1931.

Résultats de la Mission scientifique suisse en Angola, 1928-29.

\title{
Galerucini (Coleoptera Chrysomelidae) d'Angola
}

par

\section{LABOISSIERE}

Correspondant du Muséum National d'Histoire Naturelle de Paris.

Avec 6 figures dans le texte.

Les Galerucini rećueillis dans l'Angola par la Mission scientifique suisse et dont le $\mathrm{D}^{\mathrm{r}} \mathrm{A}$. Monard a bien voulu me confier l'étude, comprennent vingt et une espèces, augmentant de seize le nombre de celles que j'ai citées dans le mémoire de la Mission RohanСнавот; huit espèces sont nouvelles, neuf sont répandues dans toute l'Afrique occidentale et centrale; deux: Asbecesta robusta Weise et Monolepta missis Laboiss., n'étaient connues que de l'Afrique orientale; enfin, deux individus, par trop incomplets pour être identifiés, sont fort probablement les représentants de deux espèces inédites. Le tout est réparti entre quinze genres dont un nouveau; celui-ci a été récolté dans la région du Chimporo.

Gen. Leptaulaca Weise.

Leptaulaca fissicollis. Thомson, 1858, Arch. ent. II, p. 218. Etonga, VIII.

L. fissicollis ab. nigrotransversa. Thомson, l. c., p. 217. Ebanga, VIIII.

L. fissicollis ab. obscurata. Weise, 1902, Archiv. f. Naturg., LXVIII, p. 144. Ebanga; Etonga.

L. fissicollis ab vinula. Erichson, 1859, Stett. Ent. Zeit, XX, p. 85. Lombala, VIII; Vila da Ponte, XII.

La forme typique avec ses variétés est répandue dans toute l'Afrique occidentale et centrale.

Rev. Suisse de Zool, T. 38. 1931 
Gen. Copa Weise.

Copa delata. Erichson, 1843, Arch. f. Naturg., IX, p. 165 (Galeruca). Catumbela, VII.

Espèce très répandue, nuisible aux Cucurbitacées.

Gen. Hyperacantha Chapuis.

Hyperacantha collaris. WeIse, 1901, Deutsche Ent. Zeitschr., p. 279.

Saint Amaro, IX.

Gen. Megalognatha Baly.

Megalognatha rohani. Laboissière, 1921, in Bull. Muséum national (Paris), p. 285; - Id., 1925, Miss. Rohan-Chabot, IV, fasc. 3, p. 98, Pl. II, fig. 2.

Tous les individus recueillis ont les élytres fauves, tandis que ceux rapportés par la mission Rohan-Chabot ont ces organes jaune citron.

Kubango; Vila da Ponte, XII, en nombre.

\section{Gen. Luperodes Motschulsky.}

Luperodes quaternus. Fairmaire, 1880, in: Le Naturaliste, p. 316. S. Amaro, IX.

Majeure partie de l'Afrique; Madagascar.

Gen. Оотнесa Chapuis.

Ootheca mutabilis. Sahlberg, 1829, in thon, Ent. Arch., II, 1, p. 27, t. 2, f. 27-29 (Crioceris).

Vila da Ponte, XII; un exemplaire.

Espèce répandue dans toute l'Afrique occidentale et centrale.

Gen. Ergana Chapuis.

\section{Ergana hertigi n. sp.}

Noir; tête fauve, avec la face rembrunie, les calus surantennaires noirs; pronotum, écusson et élytres fauve testacé; abdomen testacé; antennes rousses, le premier article en majeure partie brun en dessus et les articles 5-9 rembrunis; cuisses noires; tibias testacés, 
plus ou moins rembrunis vers le sommet; tarses testacés, avec le sommet de chaque article plus sombre.

Forme robuste, en ovale court; tête finement ridée en travers et éparsement ponctuée sur le vertex qui est en outre marqué d'une impression assez large sur le milieu de la base, avec des rides obliques; antennes de la moitié de la longueur du corps, assez épaisses; le deuxième article est court, un peu moins grand que le troisième; le quatrième article est de la longueur des suivants et à peu près aussi long que les deux précédents réunis.

Pronotum transversal, une fois et demie plus large que long, à bords latéraux légèrement arrondis et convergents en avant; angles antérieurs épaissis et formant une faible saillie allongée en dehors; angles postérieurs obtus; base arrondie; surface marquée d'une ponctuation éparse, microscopique, et d'une faibleimpression sur le milieu de la longueur à proximité du bord latéral. Ecusson triangulaire, lisse.

Elytres plus larges que le pronotum, arrondis aux épaules, parallèles en arrière jusqu'au tiers postérieur et séparément arrondis au sommet; leur surface est couverte d'une ponctuation fine très profonde et assez serrée, les inter-

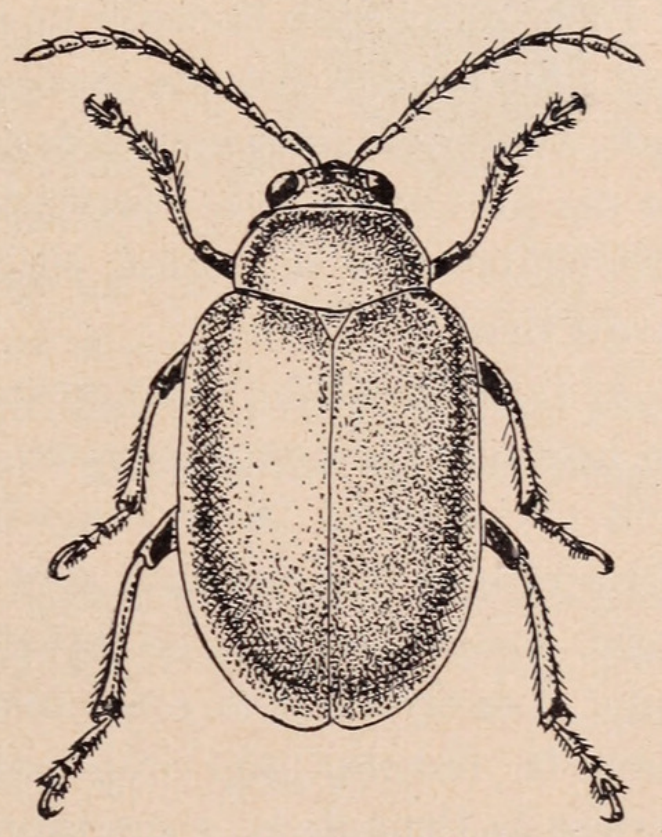

FIg. 1. - Ergana hertigi n. sp., $\times 6$. valles, un peu plus grands que le diamètre des points, sont marqués de points extrêmement fins; une faible impression longe la suture sur son premier tiers, et celle-ci paraît élevée.

Le prosternum est élevé entre les hanches qu'il sépare entièrement; il forme en arrière une saillie infracoxale sur laquelle viennent se souder les pointes épimériques; cavités cotyloïdes fermées. Les pattes sont robustes, tous les tibias sont terminés par une épine et leur sommet présente un brusque élargissement anguleux; premier article du tarse postérieur un peu plus long que les deux articles suivants réunis; ongles appendiculés. Long.: $7 \mathrm{~mm}$; larg.: $3 \mathrm{~mm}, 5$.

Vila da Ponte, XII; deux exemplaires. 
$E$. hertigi s'éloigne de toutes les autres espèces connues par sa couleur uniforme en dessus; sauf proteus Chap., qui se rencontre en Abyssinie et dont le dessus du corps est testacé, les autres espèces ont les élytres ornés de bandes longitudinales ou sont de couleur sombre; chez proteus le pronotum est éparsement et assez fortement ponctué.

Je dédie cette espèce à la mémoire du $\mathrm{D}^{\mathrm{r}}$ Hertig, membre de la Mission.

\section{Gen. Asbecesta Harold.}

Asbecesta robusta (?). Weise, 1912, Deutsche Zentr.-Afr.-Exp. IV, p. 147.

Le seul individu recueilli ne semble pas différent de ceux que j'ai reçus du Zambèze et que je considère comme appartenant à l'espèce de J. WeIsE; mais la courte description de l'auteur ne me permet pas de me prononcer avec certitude, les insectes étudiés par J. Weise provenaient de la région située au sud du lac Albert-Edouard et du Béni.

A. robusta est fauve rougeâtre clair, avec les élytres bleu bronzé ou bronzés; l'abdomen noir ou noir-bleu; les antennes et les pattes sont entièrement fauve rougeâtre ou jaunâtres.

La tête et le pronotum sont presque lisses; les antennes légèrement fusiformes ont les articles intermédiaires aussi larges que longs. Les élytres sont assez fortement et densément ponctués, avec chez la femelle une impression longitudinale commençant en arrière de l'épaule et s'arrêtant avant le milieu. Long.: $6 \mathrm{~mm}-7 \mathrm{~mm}$ (d'après J. WEISE). L'individu examiné ainsi que ceux du Zambèze ne dépassent pas $5^{\mathrm{mm}}, 5$.

Chimporo, XI, une ㅇ.

Asbecesta monardi n. sp.

Tête et pronotum testacés, labre et palpes noirs; vertex fauve; antennes testacées à la base, noires à partir du cinquième article; écusson noir; élytres bleu-vert un peu bronzé, ou bleu-violet sombre; poitrine et abdomen noirs; pattes antérieures testacées, cuisses des deux autres paires noires; tibias intermédiaires testacés, assez fortement rembrunis; les postérieurs presque noirs en dessus, plus clairs en dessous; tarses bruns.

Tête lisse; antennes filiformes, atteignant à peine le premier tiers des élytres; tous les articles, à part le second, sont un peu plus 
longs que larges; ils sont plus épais à partir du cinquième jusqu'au sommet.

Pronotum transversal, de moitié plus large que long, bords latéraux faiblement dilatés arrondis en avant du milieu; angles antérieurs droits, bien marqués, légèrement saillants; les postérieurs obtus; surface lisse, creusée audessus du milieu d'un large et profond sillon transversal, au fond duquel se trouvent quelques points; la région des angles antérieurs est égalemént finement ponctuée. Ecusson triangulaire, lisse.

Elytres beaucoup plus larges que le pronotum à la base, très faiblement dilatés en arrière; leur surface est creusée d'une ponctuation profonde et assez forte, sauf sur le calus huméral qui est lisse et un peu saillant; chez la $q$, on remarque en

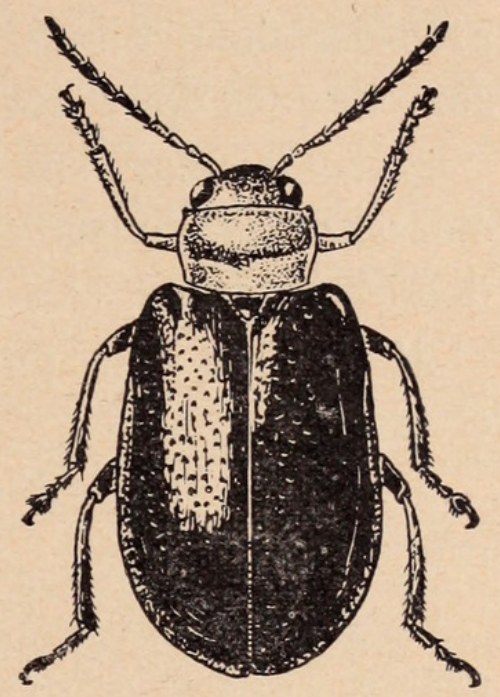

FIg. 2. - Asbecesta monardi n. sp., $\times 10$. outre une impression latérale, longitudinale, peu profonde, commençant après l'épaule et s'étendant au-delà du milieu. Long.: $3^{\mathrm{mm}}, 5-4^{\mathrm{mm}}$; larg.: $1 \mathrm{~mm}, 8-1^{\mathrm{mm}}, 9$.

o, sommet de l'abdomen trilobé; le lobe médian plan, triangulairement échancré au sommet; articles apicaux des antennes plus épais.

Tjitunda sur le Rio Cutato, I, 1929; un 今̄, une ㅇ.

Voisin de A. abdominalis Jac., mais chez celui-ci la poitrine et les pattes sont entièrement testacées.

Gen. Monolepta Erichson.

Monolepta intermedia. Ritsema, 1875, Tijdschr. Ent., XVIII“ p. 22.

Rio Mbalé; Vila da Ponte; Tjitunda.

Monolepta ludicra. Weise, 1906, Deutsche Ent. Zeitschr., p. 54. Tjitunda.

Monolepta missis nom. nov.

M. kivuensis. La zorssì̀re. 1929, Rev. zool. afric,, XVII, p, 151 fig. (non kiwuensis Weise, 1924). 
Variété: chez la forme typique la poitrine est noire et l'abdomen fauve; le seul exemplaire rapporté par la mission a la majeure partie de l'abdomen noire, seul le dernier segment est fauve.

M. missis se confond à première vue avec les deux autres espèces précédemment citées. Elles sont fauves, avec les élytres noirs et sur chaque, deux grandes taches jaunes.

$M$. intermedia se reconnaît à la couleur du dessous du corps et des cuisses fauve rougeâtre; la tache antérieure des élytres est grande et déborde le calus vers la base et en dessous.

$M$. ludicra a la poitrine et les cuisses noires; le sommet des élytres est noir.

M. missis ressemble à ludicra, mais le sommet des élytres est rougeâtre.

Chez $M$. elegans All. le sommet des élytres est également rougeâtre, mais le dessous du corps est entièrement fauve rougeâtre et les taches des élytres sont plus réduites.

Tjitunda.

Monolepta fulvofasciata n. sp.

Jaune testacé pâle; bord latéral, base et sommet des élytres

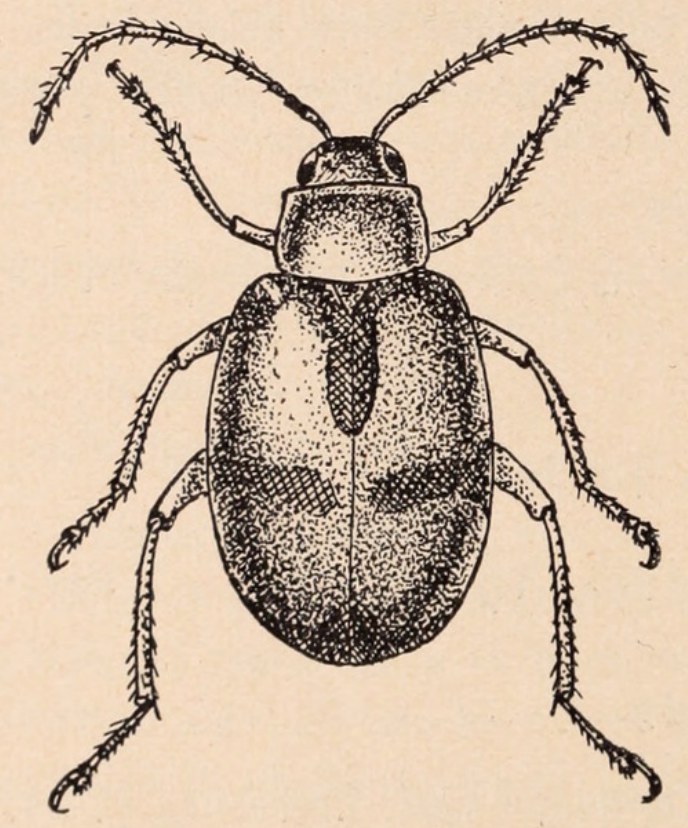

FIG. 3. - Monolepta fulvofasciata n. sp., $\times 8$. fauves ou légèrement brunâtres; une tache de la même couleur sur le calus huméral ainsi qu'une bordure suturale assez large, sur le premiers tiers, l'écusson et une étroite bande transversale obliquant légèrement du bord latéral à la suture qu'elle n'atteint pas; le rebord basal est souvent brun noir, cette couleur s'étend parfois un peu sur le début du rebord la téral; poitrine et abdomen fauves.

Ovale allongé et convexe. Tête lisse, le vertex généralement un peu plus foncé, les antennes sont filiformes, avec les articles allongés et un peu épaissis, principalement chez le $\hat{o}$, à partir du quatrième, les deuxième et troisième sont égaux. 
Pronotum de moitié plus large que long; bords latéraux droits: angles antérieurs droits, épaissis; les postérieurs obtus; la base est arrondie; surface à ponctuation extrêmement fine et peu serrée. Ecusson lisse.

Elytres densément et finement ponctués. La suture en dehors de la bande qui la suit en avant est parfois étroitement liserée de fauve pâle, mais cette couleur n'est le plus souvent visible que sur le tiers postérieur, où elle présente une pointe qui est le prolongement de la bande apicale; la bande médiane est plus sombre ainsi que la bordure basale et la tache humérale. Long.: $4 \mathrm{~mm}, 5-5 \mathrm{~mm}$.

Cette espèce se rapproche de $M$. pauperata Erichs.; mais chez cette dernière les élytres sont entourés de fauve et la bande suturale est coupée cruxialement par une bande médiane transversale, ce qui n'est pas le cas chez $M$. fulvofasciata dont la bande transversale est oblique et n'atteint pas le milieu; en plus, la suture n'est pas marginée de fauve sur son milieu.

Catumbela, trois exemplaires; je possède plusieurs individus identiques: un, provenant également de l'Angola; quatre du Gabon et un du Sénégal, environs de Dakar. Son aire de dispersion serait assez semblable à celle de $M$. pauperata .

Monolepta duplicata. Sahlberg, 1829, in Thon, Ent. Arch., II, p. 28.

Catumbela.

Cette espèce se rencontre dans la Guinée et l'Afrique centrale.

\section{Monolepia apiciventris n. sp.}

Fauve; partie supérieure de la tête, noire; face testacée; labre brunâtre; pronotum rougeâtre vif; antennes testacé pâle, le sommet des articles, à partir du cinquième, légèrement rembruni en dehors, la pointe du dernier, brune; élytres testacé pâle, avec une large bande basale et une plus étroite transversale postmédiane, noires; dessous fauve; pattes testacé pâle, l'extrême base du métatarse postérieur tachée de noir; pygidium et dernier segment abdominal noirs.

Forme ovalaire, allongée, peu convexe. Tête lisse, antennes à peine aussi longues que la moitié du corps, le quatrième article est aussi long que les deuxième et troisième réunis qui sont égaux, le cinquième article et les suivants sont moins grands que le quatrième et chacun de moitié plus long que large.

Pronotum transversal, de moitié plus large que long; bords 
latéraux faiblement divergents et arrondis de la base au milieu et plus fortement convergents en avant; tous les angles sont obtus et peu marqués; la surface très finement ponctuée est creusée sur le milieu d'une large mais peu profonde impression transversale, interrompue dans le milieu. Ecusson fauve rougeâtre, lisse.

Elytres ovalaires, allongés, très finement et peu visiblement ponctués. Long.: $3^{\mathrm{mm}}, 5$.

Une vingtaine de Monolepta africains sont testacés, avec des bandes transversales noires sur les élytres. Sur ce nombre, huit se distinguent par la tête plus ou moins noire; quelques espèces ont l'abdomen noir; il est entièrement testacé chez les autres. M. apiciventris se distinguera done facilement par le pygidium et le sommet de l'abdomen noirs et, surtout, par l'impression prothoracique; ce caractère est extrêmement rare dans le genre.

Vila da Ponte; un exemplaire.

\section{Gen. Candezen Chap.}

\section{Candezea costatipennis n. sp.}

Jaune clair ou testacé pâle; labre noir; pointe du dernier article des antennes brune; élytres parsemés de petites taches arrondies noires, généralement plus nombreuses en arrière; dernier article des palpes et ongles bruns; hanches, poitrine, abdomen et une tache à la base du métatarse postérieur, noirs.

Ovalaire, convexe. Tête assez densément et finement ponctuée; antennes filiformes, dépassant le milieu des élytres; tı oisième article de moitié plus long que le second, les suivants beaucoup plus grands.

Pronotum un peu moins de deux fois plus large que long, à bords latéraux légèrement arrondis et convergents en avant; ils sont limités en dedans par une gouttière nette et profonde; base arrondie, sinuée devant l'écusson; angles antérieurs légèrement saillants; les postérieurs obtus; surface très finement pointillée et finement ponctuée. Ecusson triangulaire, lisse; il est parfois brun à la base.

Elytres grands, ovalaires, très finement pointillés, finement et assez densément ponctués; ils sont ornés de taches généralement petites, mais irrégulières, celles placées en arrière un peu plus grandes, elles sont asymétriques, disposées différemment et en nombre inégal, variant de 15 à 30, sur chaque élytre; quand le nombre est réduit, c'est toujours sur la moitié antérieure que l'effacement se produit. 
Epipleures larges en avant, fortement rétrécis après le milieu et atteignant l'angle latéral. Long.: $6^{\mathrm{mm}}, 5-7 \mathrm{~mm}$.

$\widehat{0}$, élytres pourvus au-dessous de l'écusson et près de la suture d'une petite élévation longitudinale, ovalaire, et ornés d'une carène élevée partant du dessous de l'épaule et se dirigeant obliquement en arrière jusqu'au commencement de la déclivité latérale pour se terminer vers le milieu de la longueur. Sommet de l'abdomen fortement incisé de chaque côté, trilobé, le lobe médian très long, impressionné assez fortement sur toute son étendue.

ㅇ, élytres dépourvus d'élévation basale et de carène latérale,

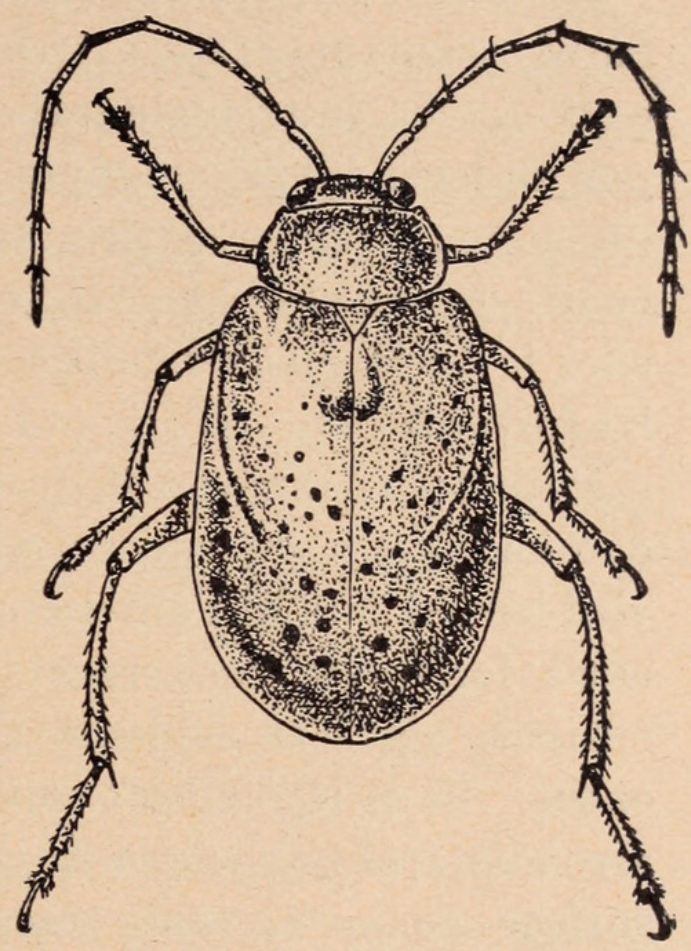

FIG. 4. - Candezea costatipennis n. sp., $\widehat{o} \times 6,6$. très légèrement impressionnés à l'emplacement de cette dernière. Sommet de l'abdomen creusé d'une petite impression longitudinale.

Vila da Ponte; Catumbela; Tjitunda quatorze exemplaires.

C. costatipènnis se confond à première vue avec $C$. irregularis Rits. qui est extrêmement commun dans l'Afrique occidentale et centrale et qui a été recueillie en Angola par la Mission Roнan-Снавот; mais chez cette espèce, de même que chez occipitalis Reiche et duvivieri Jac. dont les élytres sont ornés d'un grand nombre de taches, ceux du mâle présentent également une élévation ou fossette basale, mais sont dépourvus de carène. C. occipitalis, particulier à l'Abyssinie, a également le corps noir en dessous ainsi que la face de la tête et le sommet du vertex, la majeure partie des ántennes, etc...

\section{Сhimporia ${ }^{1} \mathrm{n}$. gen.}

En ovale court. Tête enfoncée dans le pronotum jusqu'au bord supérieur des yeux, qui sont grands, ovalaires et peu saillants;

1 Nom tiré du lieu de capture: Chimporo. 
palpes maxillaires grèles; dernier article allongé et pointu; labre transversal; clypeus peu saillant, ne présentant pas de carène nette entre les antennes; son extrémité se soude aux calus surantennaires; ceux-ci sont en triangle transversal, peu élevés et limités en dessus par un léger sillon; antennes filiformes, de la moitié de la longueur du corps; premier article faiblement épaissi; les deux suivants courts, égaux chez le $\widehat{o}$; le troisième un peu plus long que le second chez la f; les deux réunis sont moins grands que le quatrième ou les suivants.

Pronotum transversal, près de deux fois plus large à la base, que long dans son milieu; rebordé sur son pourtour, mais à peine visiblement au bord antérieur qui est droit; bords latéraux droits, assez fortement convergents vers les angles antérieurs qui sont épaissis, aigus et saillants en avant; les angles postérieurs sont obtus, vifs et légèrement saillants en arrière; la base est largement arrondie, très faiblement sinuée de chaque côté et devant l'écusson; surface convexe, sans trace d'impression. Ecusson en trianlge équilatéral.

Elytres ovalaires, étroitement rebordés sur la base et le bord latéral jusqu'à proximité de l'apex, très convexes, avec une seule impression obsolète, en dedans du calus huméral. Epipleures larges et un peu concaves en avant; ils se rétrécissent rapidement à partir du premier tiers de leur longueur et disparaissent vers l'angle latéral.

Prosternum invisible entre les hanches antérieures qui sont conoïdes et contiguës; il présente en arrière de celles-ci un petit lobe à proximité duquel s'arrêtent les pointes épimériques, les cavités cotyloïdes sont donc très faiblement entr'ouvertes; hanches médianes très rapprochées, à peine séparées par un prolongement linéaire du mésosternum. Pattes normales, tibias non sillonnés ou carénés en dessus, tous terminés par une épine, plus longue aux postérieurs; métatarse postérieur de la longueur des trois articles suivants réunis; ongles appendiculés; les cuisses intermédiaires du mâle sont échancrées sur le bord inférieur et garnies d'une rangée de longs poils; chez la temelle elles sont simplement garnies d'une rangée de poils, moins longs que ceux du mâle; les tibias postérieurs chez le mâle sont arqués.

Ce nouveau genre se place à côté des Monolepta dont il a l'aspect général; sa forme est plus robuste; le pronotum est plus large à la 
base, ses côtés plus fortement convergents en avant et les angles saillånts; le métatarse postérieur est plus robuste et moins long; enfin les cavités cotyloïdes antérieures ne sont pas complètement closes en arrière des hanches et les épipleures élytraux sont plus longs; il se sépare de Candezea par le troisième article des antennes plus long que le second dans ce dernier genre et de Barombiella caractérisé par son prosternum aussi élevé que les hanches qu'il sépare entièrement.

Le Candezea ciliata Weise, in Sјöste d т, Kilim. Exp. I, 1909, p. 213, doit appartenir à ce nouveau genre. L'auteur indique sa forme plus trapue, l'échancrure des cuisses garnies de cils, la brièveté relative du métatarse, etc.

Géno-type: Chimporia monardin. sp.

\section{Chimporia monardi n. sp.}

Fauve rougeâtre vif, antennes fauves à la base, rembrunies sur le cinquième article et noires sur les autres articles; parfois noires à partir du quatrième article; le labre est généralement jaune plus pâle, mais chez le mâle seulement; élytres fauve brunâtre, chacun avec deux grandes taches jaune vif, la première en carré transversal est située avant le milieu, la seconde, en triangle irrégulier est placée sur la seconde moitié; la partie basale, le bord latéral, la suture et la bande transversale de couleur brune sont le plus souvent recouverts sur presque toute leur étendue de noir brillant ainsi que les épipleures, le sommet reste toujours brun fauve; la poitrine est un peu plus foncée que l'abdo-

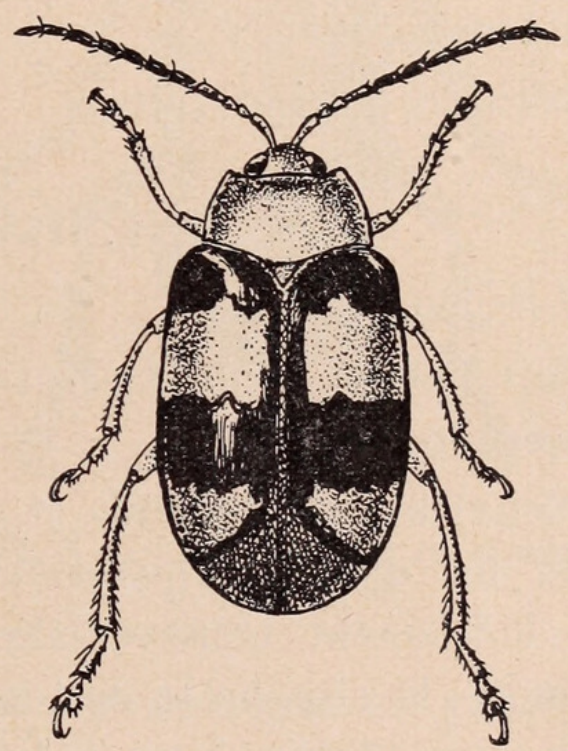

FIG. 5. - Chimporia monardi n. sp., $\times 7,5$. men chez le $\hat{o}$, et presque complètement noire chez la $q$; un point noir se trouve à la base du métatarse postérieur.

Tête lisse. Pronotum assez densément et finement ponctué. Ecusson lisse. Elytres densément et assez finement ponctués, avec les intervalles étroits et ruguleux; la ponctuation creusée au-dessus 
de la marge est beaucoup plus fine et extrêmement serrée. Long.: $5^{\mathrm{mm}}-5^{\mathrm{mm}}, 5$; larg. : $2^{\mathrm{mm}}, 5-2^{\mathrm{mm}}, 75$.

ô; cuisses intermédiaires échancrées et ciliées à leur bord inférieur; sommet de l'abdomen trilobé.

Le système de coloration rappelle les Monolepta clienta Weise, sternalis Weise, etc...; le Candezea ciliata Weise a également les élytres jaunes, entourés de fauve, avec une étroite bande médiane transversale.

Chimporo, sept exemplaires.

\section{Gen. Barombiella nom. nov. ${ }^{1}$}

Barombiella sp.

Entièrement jaune testacé pâle; antennes et pattes incomplètes.

En ovale allongé. Tête finement ponctuée. Pronotum trapézoïdal; alutacé et finement ponctué en dessus. Elytres densément et finement ponctués. Long.: $5 \mathrm{~mm}$.

Forme probablement nouvelle, mais en trop mauvais état pour être décrite.

Cakindo, un individu.

Gen. Hemixantha Jacoby.

\section{Hemixantha sp.}

Noir bronzé brillant; élytres châtain brillant; dessous garni de pubescence jaune.

Pronotum densément et profondément ponctué, marqué en outre de quatre impressions très légères placées comme suit: une faiblement transversale au-dessous du bord antérieur; une arrondie, la plus nette de toutes, devant le milieu de la base et les deux autres très petites se trouvant une de chaque côté du milieu; élytres densément et profondément ponctués. Long.: $6 \mathrm{~mm}$.

Le seul individu rapporté est totalement dépourvu d'antennes et il ne possède que les pattes postérieures privées de leurs tarses; sa couleur très particulière le sépare de tous les Hemixantha connus, genre dans lequel je crois pouvoir classer cet insecte; mais l'absence

1 En remplacement de Barombia Jac., 1903 (non Barombia Karsch, 1891, Orthoptère). Ce renseignement m'a été communiqué par le $\mathrm{D}^{\mathrm{r}} \mathrm{J}$. CARL, à qui j'adresse ici mes bien sincères remerciements, 
des principaux organes ne permet pas de me prononcer avec certitude.

Chimporo.

Gen. Platyxantha Baby.

Platyxantha (Haplotes) angolensis n. sp.

Noir brillant; palpes maxillaires, élytres et pattes jaune clair ou légèrement fauves; labre brun ou noir, marginé de pâle.

o, forme allongée, grèle, à téguments très peu consistants. Tête aussi large que le pronotum en comprenant les yeux qui sont saillants; calus surantennaires triangulaires, grands, peu convexes et finement réticulés, limités en dessus par un sillon droit; vertex finement réticulé; antennes presque aussi longues que le corps, filiformes, le deuxième article seul très petit, quatre fois moins long que le troisième, le quatrième aussi grand que les deux précédents réunis.

Pronotum à peine d'un quart plus large que long; bords latéraux parallèles sur le quart basal, puis légèrement divergents et faiblement arrondis jusqu'au bord antérieur; tous les angles bien marqués, droits, les antérieurs

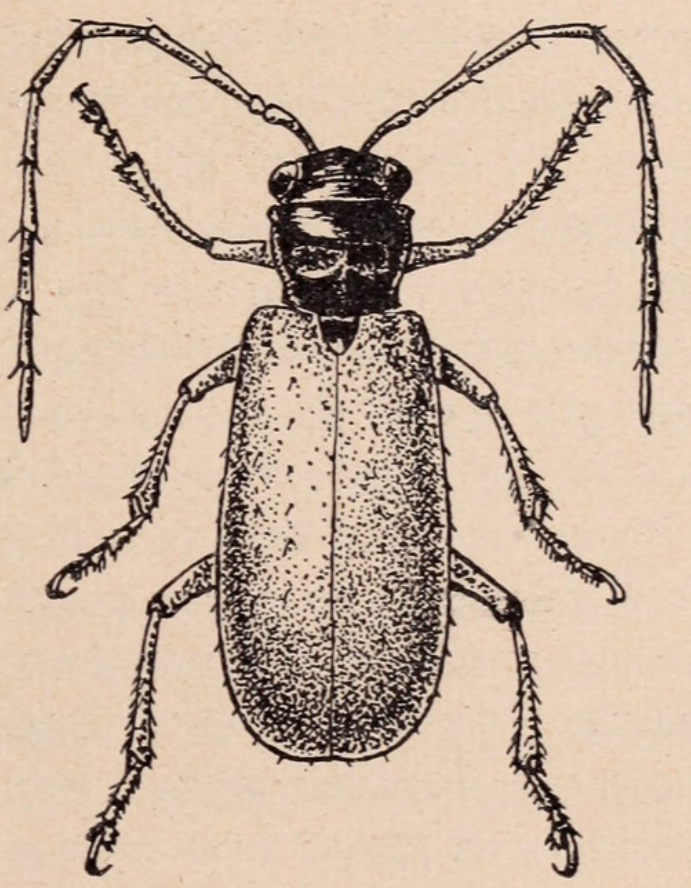

Fig. 6. - Platyxantha angolensis n. sp., $\times 6$.

saillants; surface finement réticulée et éparsement ponctuée, creusée au-dessous du milieu de deux larges impressions transversales, profondes, confluentes, mais moins marquées à leur jonction. Ecusson triangulaire, lisse.

Elytres allongés, très faiblement élargis en arrière; leur surface, peu convexe, est très finement et densément réticulée, avec quelques points fins très épars; on remarque en outre la présence de deux lignes - sans compter la marge — de poils dressés très espacés; une faible dépression limite les calus huméraux en dedans.

Pattes longues et grèles, tous les tibias mutiques au sommet; 
métatarse postérieur plus long que les deux articles suivants réunis. Long.: $7 \mathrm{~mm}$; larg.: $2^{\mathrm{mm}}, 5$.

Rio Mbalé; Tchitunda; deux 우.

$P l$. angolensis s'éloigne par sa couleur de toutes les espèces connues.

\section{Platyxantha (Haplotes) borlei n. sp.}

Dessous bleu verdâtre plus ou moins métallique; dessus bleu ou vert légèrement métallique; bouche noire; antennes fauves, parfois rembrunies au sommet; pattes fauve pâle; deux derniers articles des tarses, bruns.

Forme allongée, mais moins grèle que la précédente. Tête courte; vertex finement réticulé, marqué sur son milieu au-dessus du sillon transversal d'une impression nette; antennes moins longues que le corps, ô; atteignant le milieu des élytres, $q$; articles épaissis, pubescents, le deuxième seul petit et globuleux, le quatrième est égal au troisième chez le $\hat{o}$, et plus long chez la ․ Chez celle-ci tous les articles sont cylindriques et chacun environ deux fois plus long que large; ils sont plus développés dans le mâle, arrondis en dessus et échancrés au bord inférieur à partir du sixième.

Pronotum d'un tiers plus large que long; bords latéraux faiblement dilatés-arcondis en avant; angles petits, peu marqués, les antérieurs droits, les postérieurs légèrement obtus; surface peu convexe, densément et finement réticulée, subalutacée, creusée au-dessous du milieu de deux impressions larges, en ovale transversal contiguës et moins profondes sur le milieu. Ecusson triangulaire arrondi au sommet, lisse.

Elytres plus larges que le pronotum à la base, recouverts d'une fine réticulation et éparsement ponctués, légèrement impressionnés en travers sur le premier tiers.

Pattes assez robustes, tous les tibias mutiques; métatarse postérieur de la longueur des deux articles suivants réunis. Long.: $5 \mathrm{~mm}$.

$P l$. borlei se place à côté de ruxvenzorica Weise; ce dernier est plus grand, avec les antennes et les pattes sombres.

Kubango; un $\widehat{o}$, une q.

Je dédie cette espèce à MM. BorLe, père et fils, membres de la mission. 


\section{$2 \mathrm{BHL}$ Biodiversity Heritage Library}

Laboissière, V. 1931. "Galerucini (Coleoptera Chrysomelidae) d'Angola." Revue suisse de zoologie 38, 405-418. https://doi.org/10.5962/bhl.part.117646.

View This Item Online: https://www.biodiversitylibrary.org/item/148559

DOI: https://doi.org/10.5962/bhl.part.117646

Permalink: https://www.biodiversitylibrary.org/partpdf/117646

\section{Holding Institution}

American Museum of Natural History Library

\section{Sponsored by}

BHL-SIL-FEDLINK

\section{Copyright \& Reuse}

Copyright Status: In copyright. Digitized with the permission of the rights holder.

Rights Holder: Muséum d'histoire naturelle - Ville de Genève

This document was created from content at the Biodiversity Heritage Library, the world's largest open access digital library for biodiversity literature and archives. Visit BHL at https://www.biodiversitylibrary.org. 